\title{
Theoretical and experimental characterization of a FEM element assembly for the simulation of very compliant knitted mesh
}

\author{
Michele Falcone, Emanule Marotta, Ettore Pennestrì, Pietro Salvini, Pier Paolo Valentini \\ University of Rome "Tor Vergata" \\ Department of Enterprise Engineering \\ Via del Politecnico, 1 - 00133 Rome, ITALY \\ Email: valentini@ing.uniroma2.t
}

\begin{abstract}
The paper deals with the description, development and validation of an equivalent FEM element assembly suitable for the simulation of very compliant knitted meshes like those used in elastic coatings, deployable antennas, medical applications and similar. The assembly is based on a network of lumped springs able to reproduce the orthotropic behaviour of the mesh when stretched along two directions. The parameters of the model have been identified by experimental tests using a dedicated biaxial testing device. The model has been implemented for two different types of tests in order to assess the validity for both in-plane and outof-plane displacements. The matching between numerical results and experiments is very good and the differences are bounded below $6 \%$ for deformations up to $50 \%$.
\end{abstract}

Keywords: knitted mesh, finite element modelling, structural stiffness, biaxial stretching

\section{Introduction}

Due to the increasing interest in smart materials, the role of very compliant knitted meshes is gaining an important attention in many different fields. A generic mesh is made of several knits, repeated in an ordinate and periodic structure. Knits may or may not present symmetries. Each knits is comprised of yarns of different pattern. These types of structure are increasingly used in different applications. For example, metallic knitted meshes can be used in the development of sensors and antennas [1]. Elastomeric meshes can be used for improving bandages [2], surgical devices [3] or biomedical coatings [4]. Mixed elastomeric and fabric meshes 
can be used as technical [5] or smart cloths [6]. In all these applications, it is important an accurate assessment of the elastic properties of the materials since they highly influence the performance of the final product. In most of the cases, due to the particular knitting pattern, the elastic behaviour is nonlinear and orthotropic and the meshes undergo very large displacements with consequent large deformations and rearrangement of all the internal knits. The characterization of these properties is not a simple task. In most of the cases, they are assessed with mono-axial stretching tests [7]. Due to the specific behaviour of the meshes [8], in some other cases, bi-axial test machines have been used [9-10]. The main difficulties in these measurements are represented by the very low global stiffness and very large displacement of the meshes that require specific modifications of the standard testing equipment. Moreover, due to the compliance of the knitting pattern, the clamping system may introduce boundary stiffening effects which alter the elasticity assessment [11].

The complete assessment of the elastic properties is mandatory for the setup and characterization of a reliable mathematical model able to simulate and predict the complex behaviour of the mesh. Such a model can be useful for design purposes and optimizations.

An analysis of the scientific literature reveals that the research has been mainly focused on fabric meshes. In many cases, the research is aimed toward an efficient model for simulating the dynamics of the garments but there is not a systematic approach to identify the physical parameter of the model [12-16]. Most of the investigations are focused on computationally efficient models which can yield a realistic behaviour not completely suitable for an engineering point of view. Furthermore, fabric meshes are subjected to lower stretching deformation and the achievement in modelling such component is often not suitable for very compliant behaviour.

Other papers, more focused on engineering point of view, present more complex mathematical models of the mesh suitable for finite element implementation [17-19]. Some of them introduce interesting plate or laminate elements [20], which include material nonlinearities [21] and, in some cases, even geometrical nonlinearities [22]. The contribution of geometrical nonlinearities appear fundamental in meshes undergoing very large deformations due to the important deformation of the knits [23-24]. 
In order to be suitable for engineering purposes, all these models have to be characterized by the identification of all their physical parameters. In general, complex plate elements are difficult to be characterized due to the interdependence of their parameters and it is not simple to take into account both material nonlinearity and geometrical deformation of the knits. For this purpose, some authors have proposed physical models made of lumped springs [25]. The advantages of these approaches are:

- Model simplification;

- Easy parameter identification;

- Little computational effort required also for the case of large models.

- Capability to introduce non-linearities on the stiffness properties of the lumped springs.

As mentioned before, the measurement devices and protocols and the related mathematical models cannot be directly applied to very compliant mesh with a very low-stiffness. Many authors have proposed mathematical formulations based on the characterization of a plate element with specific material properties. This approach allows to include material nonlinearity, but neglects the geometrical nonlinearities due to the rearrangement of the knits [17].

Starting from this background, the purpose of the paper is to discuss a novel mathematical model suitable for finite element method implementation and aimed to describe the structural elasticity of a very compliant mesh. The model is identified through experimental measurements on a metallic mesh, performed on a custom-made testing machine. The machine can perform bi-axial tests while limiting the effect of boundary clamping.

The paper is organized as follows. In a first part, the mathematical model of the mesh element assembly is introduced. Then, the experimental setup and measurements are discussed presenting typical force/displacement plots for very compliant metallic mesh. In a subsequent section, examples of applications and validation are presented focusing on the direct capability to successfully simulate out-of-plane behaviour. 


\section{Mathematical model}

The proposed model of a mesh is depicted in Figure 1. It includes four boundary nodes (1, 2, 3 and 4), four mid-side nodes $(5,6,7$ and 8$)$, which are symmetrically connected by several spring elements. Ten springs account for the compliance along the main $x$ and $y$ directions (five springs for each direction). Four other springs are oriented diagonally and their contribution to the horizontal and vertical stiffness depends on their attitude angle (i.e. the ratio between $x$ and $y$ projections, angle $\alpha$ later in the paper). This arrangement portrays both generic compliance and non-linear geometrical behaviour due to the alignment of the fibres during the deformation. Following this approach, the element assembly is independent from the actual knitting pattern, but it can be suitable for describing a variety of possible solutions after the identification of all the elastic parameters.

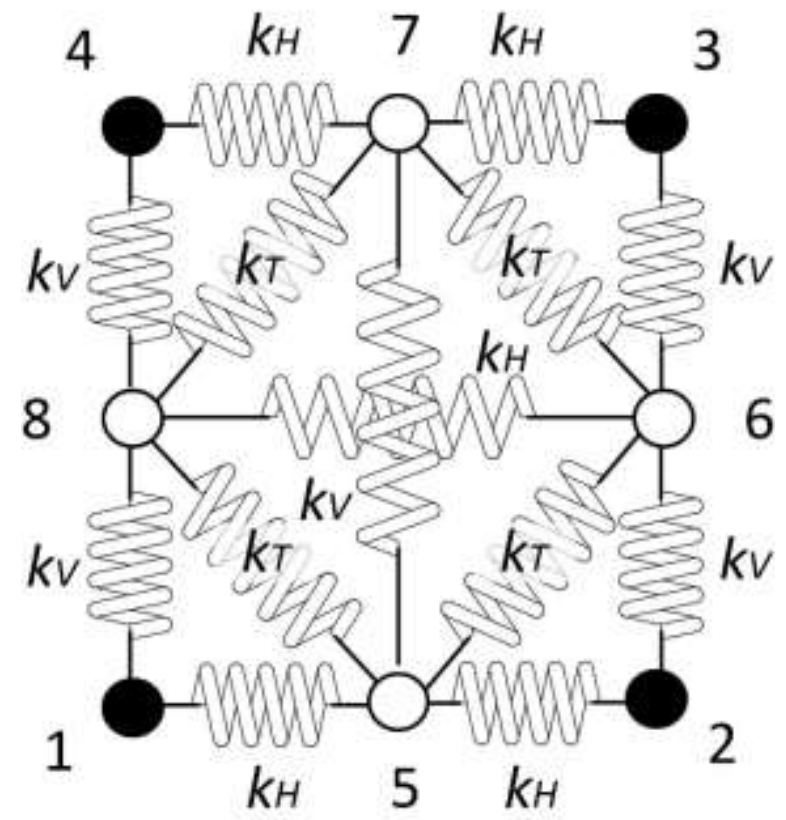

Figure 1. The proposed basic element assembly for the description of the knitted mesh

The element assembly is symmetric, thus the independent stiffness parameters to be identified are the following:

$k_{H}$ is the stiffness parameter of the elements along the horizontal direction (5 elements); 
$k_{V}$ is the stiffness parameter of the elements along the vertical direction ( 5 elements);

$k_{T}$ is the stiffness parameter of the elements along the diagonal direction (4 elements);

The deformation of the element assembly is characterized with the following four quantities:

$\varepsilon_{H}=\frac{l_{H}-l_{H 0}}{l_{H 0}}$ is the deformation in the horizontal direction $\left(l_{H}\right.$ is the horizontal length of the element assembly after the deformation and $l_{H 0}$ is the horizontal length when undeformed);

$\varepsilon_{V}=\frac{l_{V}-l_{V 0}}{l_{V 0}}$ is the deformation in the vertical direction $\left(l_{V}\right.$ is the horizontal length of the element assembly after the deformation and $l_{V 0}$ is the horizontal length when undeformed);

$\varepsilon_{T}=\frac{l_{T}-l_{T 0}}{l_{T 0}}$ is the deformation in the diagonal direction $\left(l_{T}\right.$ is the diagonal length of the element assembly after the deformation and $l_{T 0}$ is the diagonal length when undeformed);

$\alpha=\operatorname{atan}\left(l_{H}\left(1+\varepsilon_{H}\right), l_{V}\left(1+\varepsilon_{V}\right)\right)$ is the internal angle (attitude of the transverse springs) measured from the horizontal direction.

All the stiffness parameters can be identified by means of experimental biaxial-tests according to the procedure discussed in the following section.

\section{Experimental tests on the knitted mesh}

The experimental tests on different types of meshes have been performed with a custom made equipment, which has been designed and assembled in the department laboratory (Figure 2). The device includes a frame in which a portion of mesh can be mounted. The mesh is grabbed using adjustable hooks and each edge can slide within very low-friction rolling guides. The equipment is able to apply an enforced and independent displacement along $x$ and $y$ directions of the mesh and measure the corresponding resisting forces. The 
displacement is applied trough a wire and pulleys system connected to two stepper motors. Loads are measured by means of four load cell extensometers. The equipment is also controlled and monitored by a computer unit in order to apply the programmed testing conditions, manage the data logging and storage.

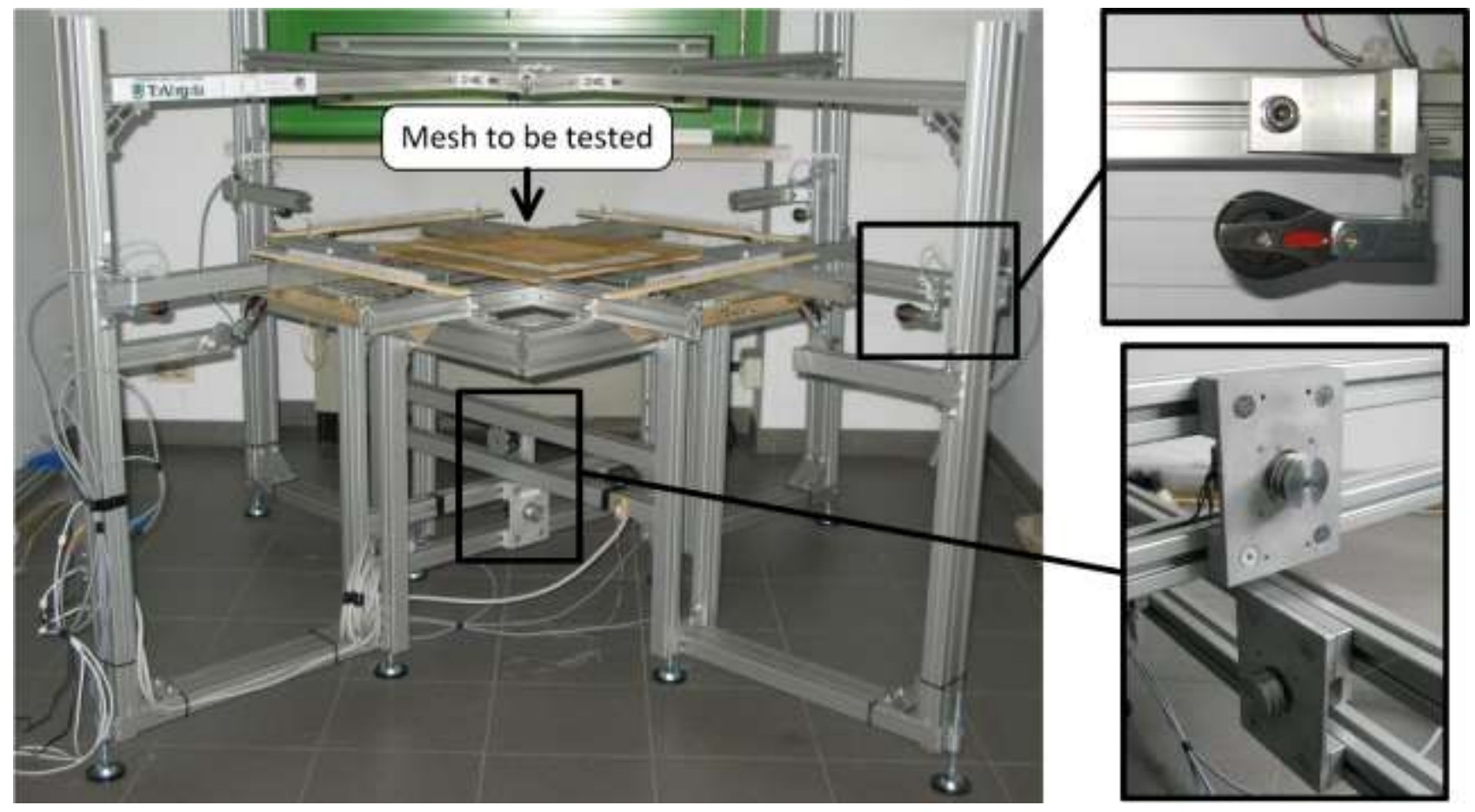

Figure 2. The experimental bi-axial test rig

The equipment is able to test different size of mesh from $10 \times 10 \mathrm{~mm}$ to $400 \times 400 \mathrm{~mm}$ and can produce large displacements (up to $400 \mathrm{~mm}$ each direction). If different types of mesh with different load capacity need to be tested, different load cells and a new calibration of the device is required. For the purpose of the paper, the investigation focuses on metallic meshes.

Although the equipment is fully programmable, three types of tests have been performed on the meshes (see schematics in Figure 3). All the measurements start with an applied preload in both $x$ and $y$ directions able to reduce the vertical inflexion to $1 / 50$ of the length of the mesh.

The first two measurements are the controlled stretching along $x$ and $y$ directions, keeping the opposite one fixed. The enforced displacement consists of fixed-step increments performed slowly (about $1 \mathrm{~mm}$ per second) in order to avoid dynamic contributions. Both $x$ and $y$ reaction forces are collected during the measurements. 
The third measurement is the simultaneous controlled stretching along $x$ and $y$ directions, with equal and fixed-step increments. Again, both $x$ and $y$ reaction forces are collected during the measurements.
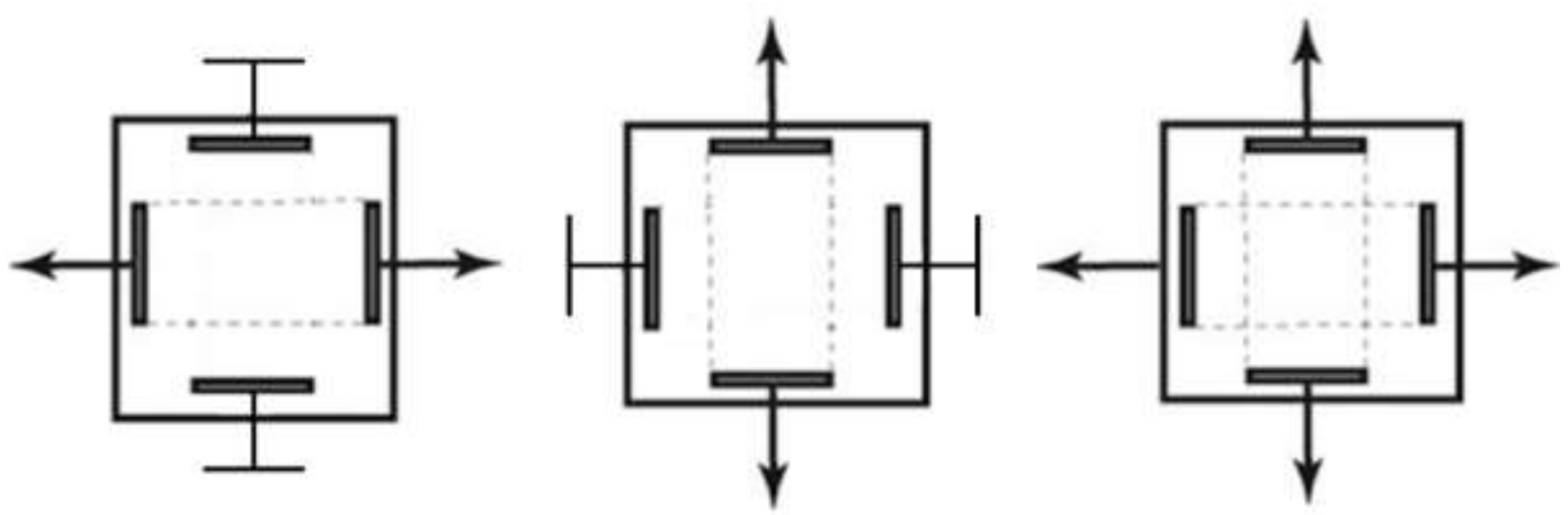

Figure 3. Schematics of the three tests performed on the mesh

The results of the tests can be plotted as surface graphs considering the deformations in $x$ and $y$ directions as the independent variables $\left(\varepsilon_{H}\right.$ and $\left.\varepsilon_{V}\right)$ and the forces as the dependent one. Figure 4 reports an example of these graphs for a metallic knitted mesh.
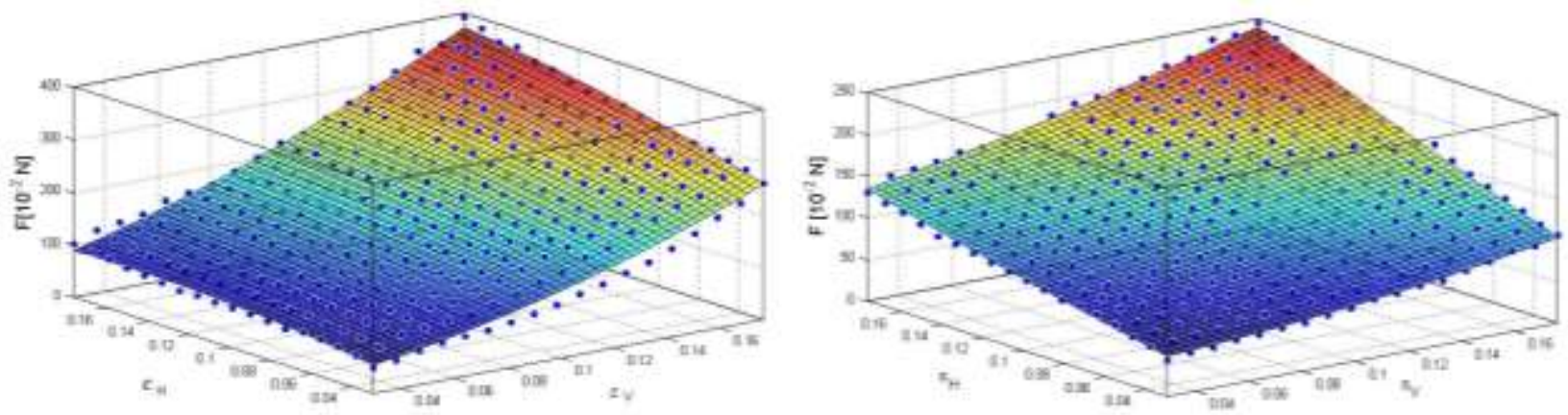

Figure 4. Force/deformation relationships for a metallic mesh. Blue dots are the experimental acquisitions; colour surface is the biquadratic surface approximation. Both refers to the first two types of measurement (stretching with constrained opposite direction). Horizontal force $F_{H}$, for an horizontal stretching is plotted on the left and vertical force $F_{V}$ for a vertical stretching on the right. The anisotropic behaviour of the mesh is clearly visible. 
It can be observed that all the force/displacement relationships can be approximated by a biquadratic surface of the form ( $b_{i}$ are the interpolation coefficients):

$$
F=b_{0}+b_{1} \varepsilon_{H}+b_{2} \varepsilon_{V}+b_{3} \varepsilon_{H}^{2}+b_{4} \varepsilon_{H} \varepsilon_{V}+b_{5} \varepsilon_{V}^{2}
$$

Errors in the approximation using biquadratic surface are within $3 \%$ and $5 \%$ of the maximum load.

Starting from the knowledge of the force functions $F=F\left(\varepsilon_{H}, \varepsilon_{V}\right)$, it is possible to compute the stiffness functions by differentiation. In particular, the differential of the $F_{H}$ (horizontal force) and $F_{V}$ (vertical force) leads to:

$$
\begin{aligned}
& d F_{H}=\frac{\partial F_{H}}{\partial D_{H}} d D_{H}+\frac{\partial F_{H}}{\partial D_{V}} d D_{V} \\
& d F_{V}=\frac{\partial F_{V}}{\partial D_{H}} d D_{H}+\frac{\partial F_{V}}{\partial D_{V}} d D_{V}
\end{aligned}
$$

where:

$$
\begin{aligned}
& \frac{\partial F_{H}}{\partial D_{H}}=-K_{H H} \\
& \frac{\partial F_{H}}{\partial D_{V}}=-K_{H V} \\
& \frac{\partial F_{V}}{\partial D_{V}}=-K_{V V} \\
& \frac{\partial F_{V}}{\partial D_{H}}=-K_{V H}
\end{aligned}
$$

$D_{H}$ and $D_{V}$ are the horizontal and vertical displacements, respectively;

$K_{i j}$ is the generic stiffness function relating the direction of force and displacement.

All functions stiffness/deformation are properly described by bilinear surfaces. Figures 5 and 6 show examples of the computation of these interpolation functions for the forces depicted in Figure 4 . The interpolated surfaces have been compared to the experimental measurements obtained with incremental load application. 

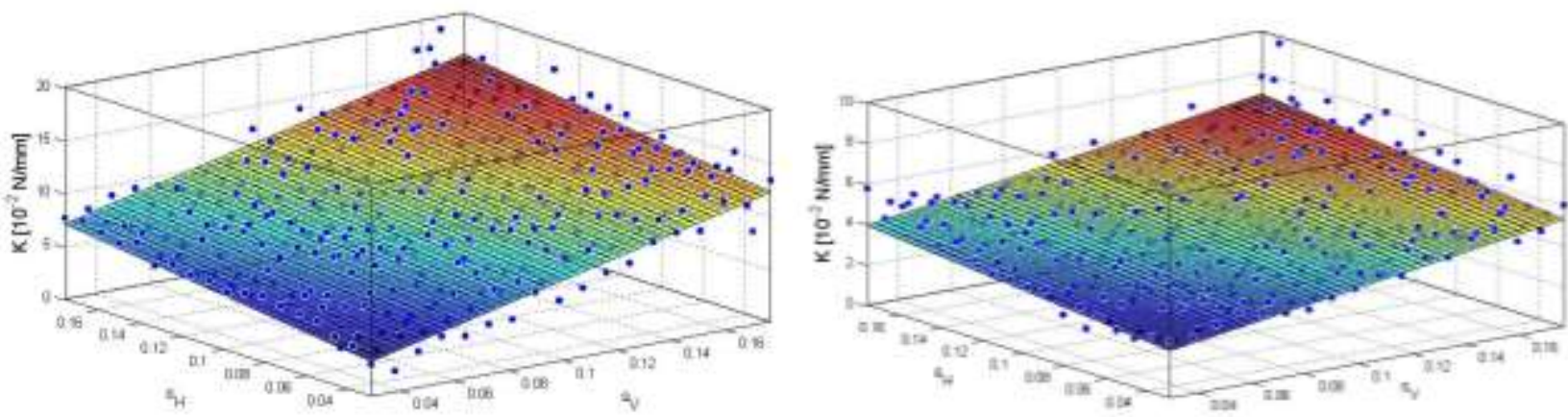

Figure 5. $K_{H H}$ (on the left) and $K_{V V}$ (on the right) stiffness/deformation relationships for a metallic mesh.

Colour surfaces are obtained by differentiation according to Eq. (3) and the blue dots are the corresponding experimental acquisitions.
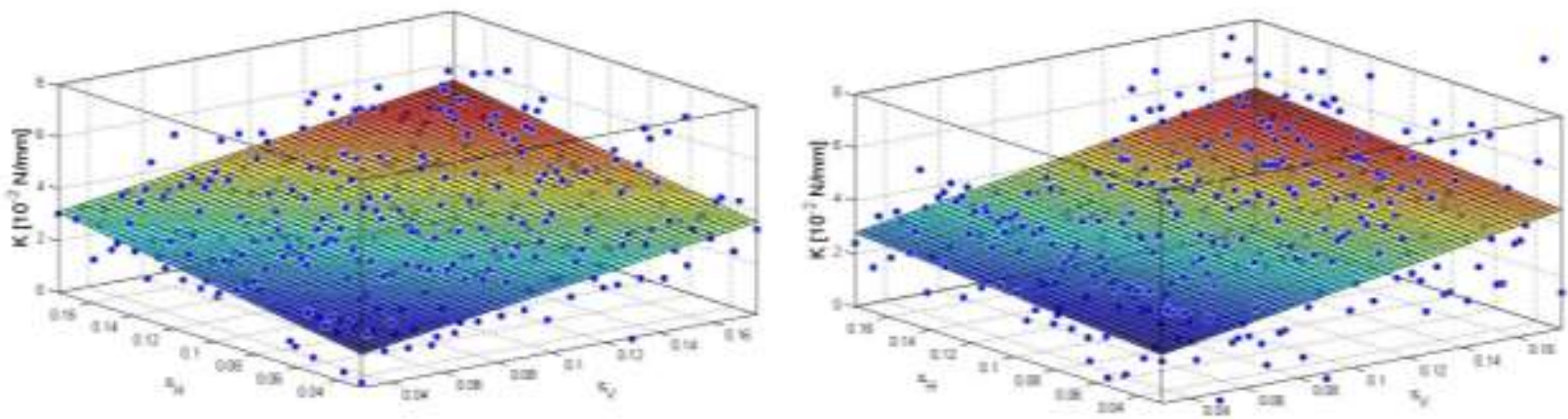

Figure 6. $K_{V H}$ (on the left) and $K_{H V}$ (on the right) stiffness/deformation relationships for a metallic mesh.

Colour surfaces are obtained by differentiation according to Eq. (3) and the blue dots are the corresponding experimental acquisitions. According to the property of symmetry of the stiffness parameters the two functions are very close.

From an experimental point of view, the $K_{V H}$ and $K_{H V}$ stiffness/deformation relationships may be computed with the third series of measurement involving the simultaneous controlled stretching along $x$ and $y$ directions, with equal and fixed-step increment $d D$ (third type of measurement of Figure 3). In this case, the Eq. (2) can be written as: 


$$
\begin{aligned}
& d F_{H}=K_{H H} d D+K_{H V} d D=K_{H H, I S O} d D \\
& d F_{V}=K_{V H} d D+K_{V V} d D=K_{\mathrm{VV}, I S O} d D
\end{aligned}
$$

Where the $K_{H H, I S O}$ and $K_{V V, I S O}$ are the stiffness/deformation relationship measured with the simultaneous controlled stretching along $x$ and $y$ directions.

The unknown values can be then computed as:

$$
\begin{aligned}
& K_{H V}=K_{H H, I S O}-K_{H H} \\
& K_{V H}=K_{V V, I S O}-K_{V V}
\end{aligned}
$$

According to Eq.(5), the $K_{V H}$ and $K_{H V}$ can be computed by the subtraction between the stiffness parameters computed with the simultaneous $x$ and $y$ displacements and those computed by single direction displacements.

\section{Identification of the element assembly parameters}

The identification of the parameters $k_{H}, k_{V}$ and $k_{T}$ can be performed through the processing of the measurements obtained during the experimental tests.

The test equipment is able to monitor the global increment of reaction forces $\left(\Delta F_{H}\right.$ and $\left.\Delta F_{V}\right)$ of the mesh along the two main directions when incremental displacements $\left(\Delta D_{H}\right.$ and $\left.\Delta D_{V}\right)$ are applied. Due to the symmetry of the mesh knitting, the incremental forces $\left(\Delta f_{H}\right.$ and $\left.\Delta f_{V}\right)$ and displacements $\left(\Delta d_{H}\right.$ and $\left.\Delta d_{V}\right)$ applied to the assembly can be computed as follows:

$$
\begin{gathered}
\Delta f_{H}=\Delta F_{H} \frac{l_{H 0}}{L_{H 0}} \\
\Delta f_{V}=\Delta F_{V} \frac{l_{V 0}}{L_{V 0}}
\end{gathered}
$$




$$
\begin{aligned}
& \Delta d_{H}=\Delta D_{H} \frac{l_{H 0}}{L_{H 0}} \\
& \Delta d_{V}=\Delta D_{V} \frac{l_{V 0}}{L_{V 0}}
\end{aligned}
$$

where $L_{H 0}$ and $L_{V 0}$ are the global horizontal and vertical length of the entire mesh, respectively.

Similarly, the stiffness coefficients of a single element assembly are related to the stiffness coefficients of the global meshes by the same constant ratio $l / L$ of the corresponding direction.

Considering a displacement increment $\Delta d_{H}$ along the horizontal direction, we can write the balance of the forces in the assembly as:

$$
\Delta d_{H} \rightarrow\left\{\begin{array}{c}
\Delta f_{H}=-2\left(k_{H} \Delta d_{H}\right)-2 k_{T} \cos ^{2} \alpha \frac{\Delta d_{H}}{2} \\
\Delta f_{V}=-2 k_{T} \sin \alpha \cos \alpha \frac{\Delta d_{H}}{2}
\end{array}\right.
$$

For a displacement increment $\Delta d_{V}$ along the horizontal direction, we can write:

$$
\Delta d_{V} \rightarrow\left\{\begin{array}{c}
\Delta f_{H}=-2 k_{T} \sin \alpha \cos \alpha \frac{\Delta d_{V}}{2} \\
\Delta f_{V}=-2\left(k_{V} \Delta d_{H}\right)-2 k_{T} \sin ^{2} \alpha \frac{\Delta d_{V}}{2}
\end{array}\right.
$$

Extracting the stiffness coefficients in both (10) and (11) we can write:

$$
\begin{aligned}
& k_{H H}=2 k_{H}+2 k_{T} \cos ^{2} \alpha \\
& k_{V V}=2 k_{V}+2 k_{T} \sin ^{2} \alpha \\
& k_{H V}=k_{V H}=k_{T} \cos \alpha \sin \alpha
\end{aligned}
$$

Eq. (12) can be rearranged in a matrix form and then solved as:

$$
\left\{\begin{array}{l}
k_{H} \\
k_{V} \\
k_{T}
\end{array}\right\}=\left[\begin{array}{ccc}
2 & 0 & \cos ^{2} \alpha \\
0 & 2 & \sin ^{2} \alpha \\
0 & 0 & \cos \alpha \sin \alpha
\end{array}\right]^{-1}\left\{\begin{array}{c}
k_{H H} \\
k_{V V} \\
k_{T T}
\end{array}\right\}
$$




\section{Model Validation}

Three tests of validation have been performed by implementing and solving two different numerical models using ANSYS finite element computer program.

The first one is about a planar rectangular mesh (see Figure 7), stretched in both $x$ and $y$ directions. The mesh is composed of 400 element assemblies (20 each side) and the rectangular shape is obtained by a ratio $l_{H 0} / l_{V 0}>1$.

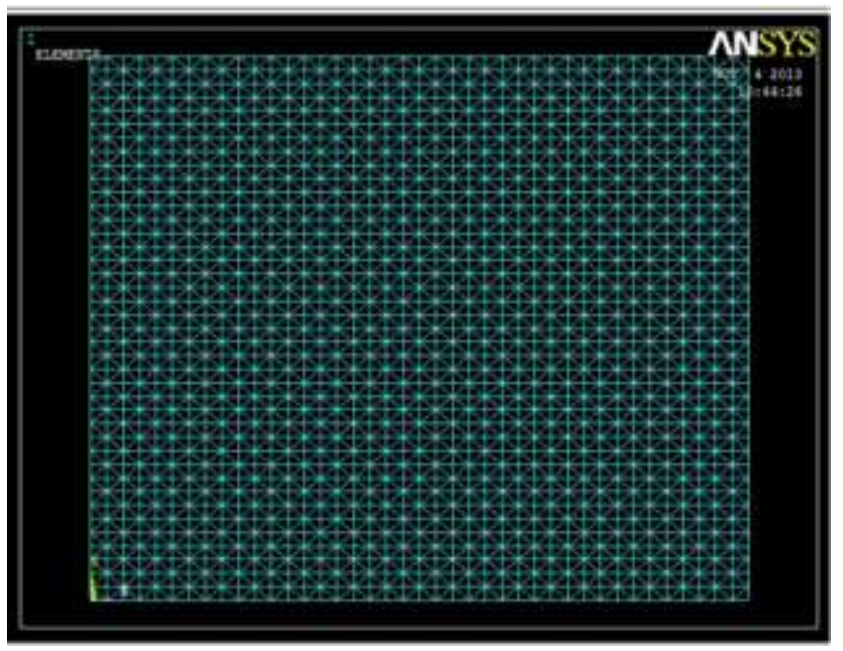

Figure 7. Numerical finite element model for the validation of the proposed method with a rectangular mesh of 400 element assemblies.

The numerical results have been compared to the corresponding experimental measurements performed at the bi-axial machine of Figure 2, in terms of horizontal and vertical forces. The results of the comparisons are reported in Figure 8. 

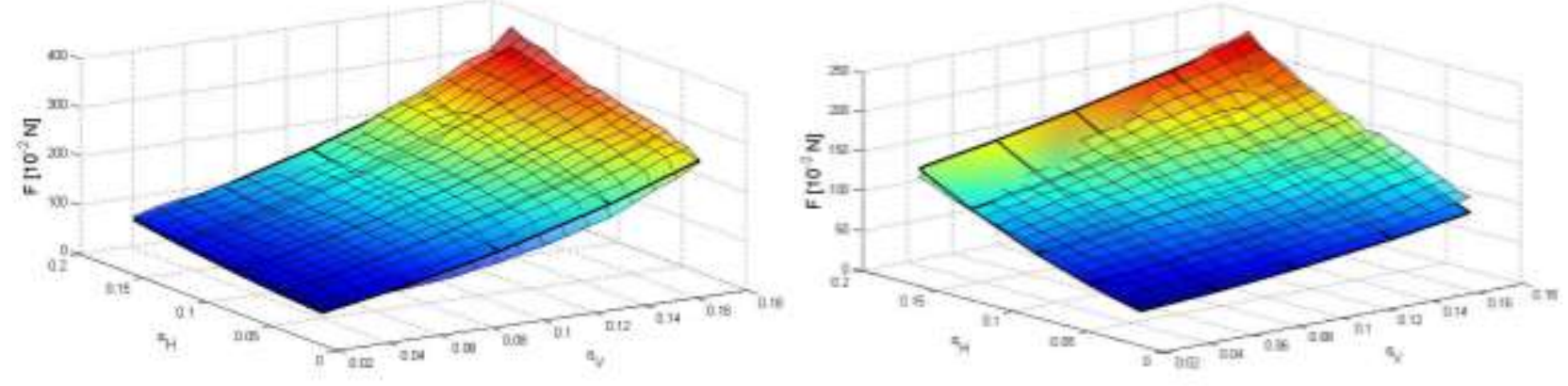

Figure 8. Comparison between numerical and experimental results on the rectangular mesh in terms of $F_{H}$ and $F_{V}$. The surfaces with smaller facets and thinner edges are those obtained by the numerical model and those with larger facet and bold edges are obtained by the measurements.

It can be noticed a satisfactory agreement for the entire range of stretching, both for linear and non linear ranges. Moreover, the not uniform behaviour along the two stretching directions is also well approximated. Maximum error is within the range of 5-6\%.

A second validation test has been performed in order to investigate the behaviour of the model in presence of an out-of-plane displacement. It is about a fully three dimensional mesh, loaded with a rigid hemisphere in the middle (see Figure 8) [26-27].

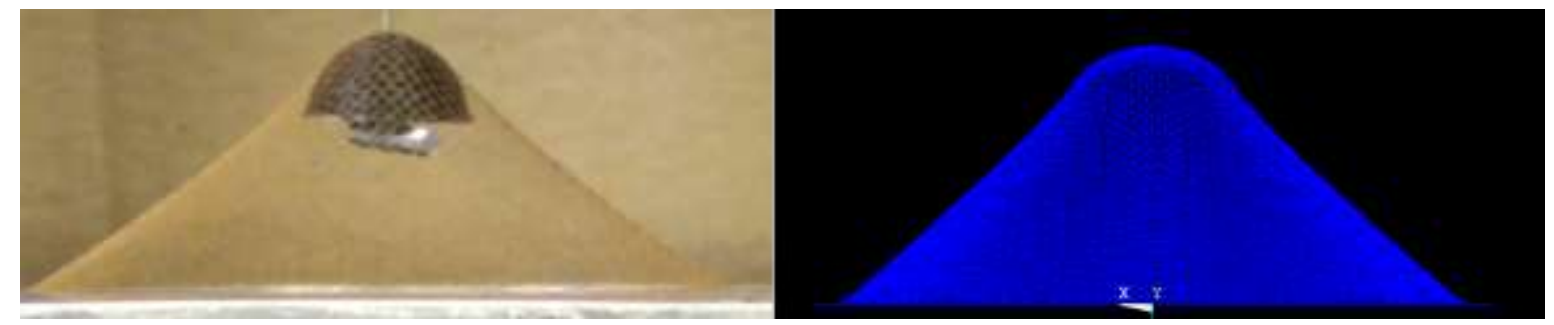

Figure 8. Validation of the proposed method considering out-of-plane displacement. The experimental measurement (on the left) and the corresponding numerical model (on the right)

The results of the model have been compared with those coming from a specific experimental campaign performed on a different device. The mesh is now rigidly fixed at the base to a frame. A spherically shaped rigid body is connected by means of a rigid wire to a mono-axial testing device able to acquire the 
force/displacement relationship (Figure 9). In particular, hemispheres with two different radii (25 and $35 \mathrm{~mm}$ ) have been used in the tests.

Only the reaction force of the sphere perpendicular to the plane of the mesh have been measured and compared. Figure 10 reports the comparison between the results of numerical model and those from experiments. In all the numerical tests, friction has been neglected. It is meaningful to observe that the two sets of results are very close, especially for large displacements where the differences are lower than $1 \%$. In the range of small displacement, there are some differences probably due to the initial stabilization of the mesh and slip with friction of the knits. Numerical results are consistent with measurements.
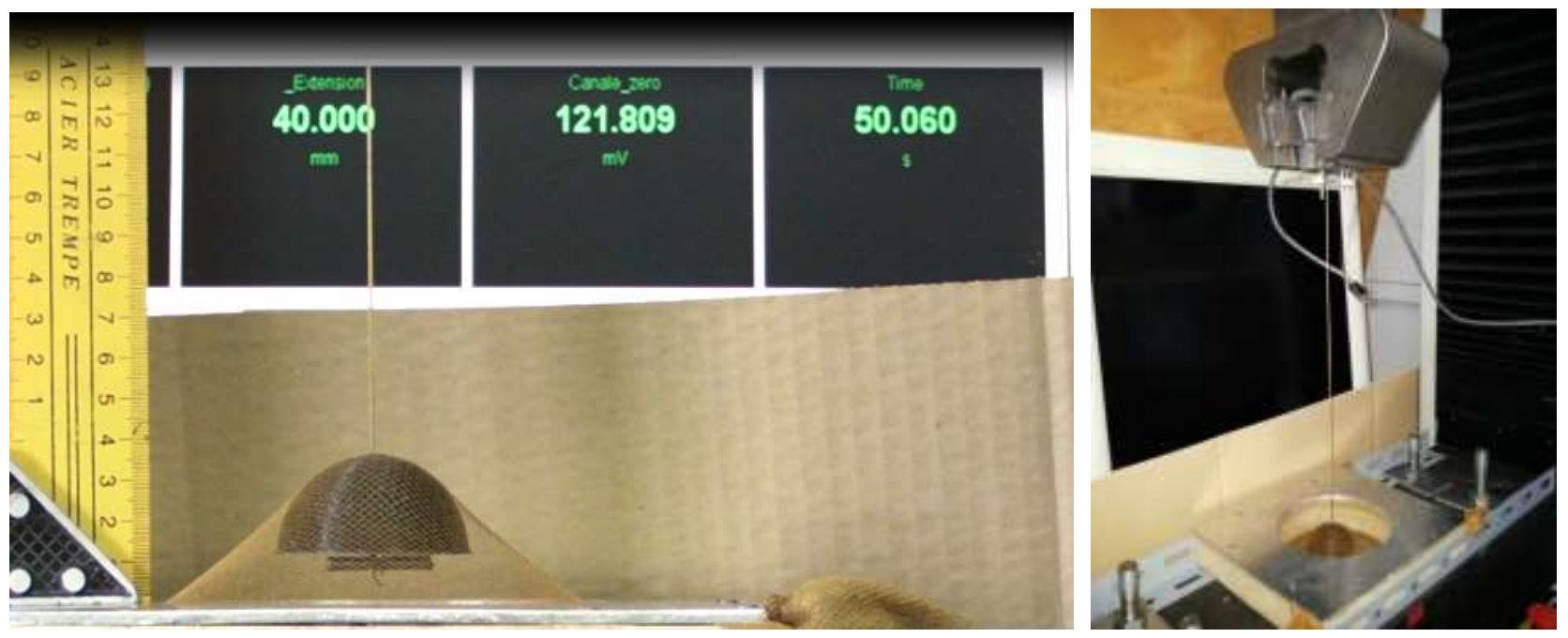

Figure 9. The experimental setup for testing the out-of-plane displacement of the knitted mesh. 


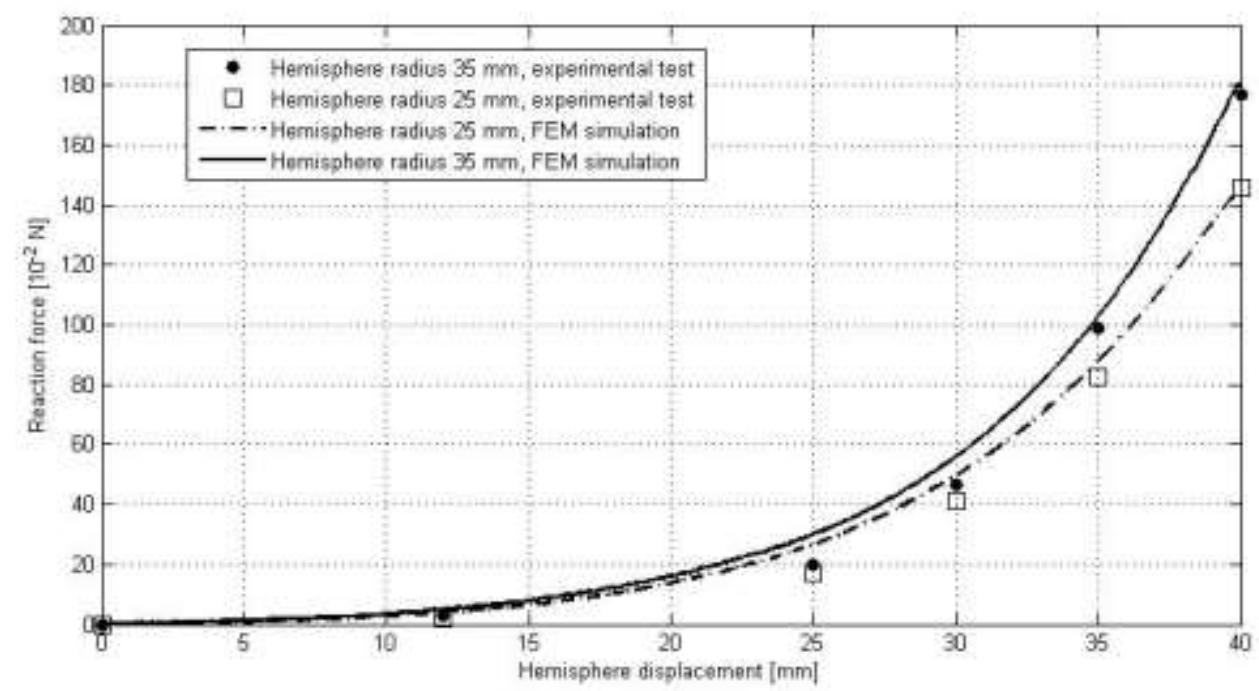

Figure 10. Comparison between simulated and measured reaction forces on the hemisphere for the validation of the out-of-plane behaviour of the knitted mesh.

\section{Conclusions}

A node-spring finite element assembly for describing the elastic behaviour of a knitted mesh has been presented. The proposed model is able to describe the nonlinear orthotropic behaviour of the mesh with a limited complexity. The model includes a collection of four boundary nodes, four internal nodes and 14 springs. Thanks to the specific arrangement of the springs, the model is also able to take into account the geometrical stiffening effect, typical of the deformation of the knits. A methodology for the identification of all the model parameters has been also discussed focusing on a custom made biaxial testing device designed for the specific purpose. The low-complexity of the model facilitate the implementation and solution of large finite element simulation with a good computational efficiency. The model has been characterized and assessed for a metallic mesh, considering both in-plane stretching and out-of-plane displacements. The consistency between numerical simulations and experimental tests is feasible for engineering purposes. Due to the typology of the model, it is also suitable for being applied to composite or elastic meshes with nonlinear structural stiffness. The parameters of such model can be identified using the same biaxial testing machine but choosing a different force-displacement interpolation surface. 


\section{References}

[1] T. Li, J. Su, Electrical properties analysis of wire mesh for mesh reflectors, Acta Astronautica, Vol. 69(1-2), pp. 109-117, 2011.

[2] G. Mosti, H. Partsch, Bandages or Double Stockings for the Initial Therapy of Venous Oedema? A Randomized, Controlled Pilot Study, European Journal of Vascular and Endovascular Surgery, Vol. 46(1), pp. 142-148, 2013.

[3] P. Feindt, U. Boeken, J.D. Schipke, J. Litmathe, N. Zimmermann, E. Gams, Ventricular constraint in dilated cardiomyopathy: A new, compliant textile mesh exerts prophylactic and therapeutic properties, The Journal of Thoracic and Cardiovascular Surgery, Vol. 130(4), pp. 1107.e1-1107.e11, 2005

[4] S.L. Edwards, J.A. Werkmeister, A. Rosamilia, J.A.M. Ramshaw, J.F. White, C.E. Gargett, Characterisation of clinical and newly fabricated meshes for pelvic organ prolapse repair, Journal of the Mechanical Behavior of Biomedical Materials, Vol. 23, pp. 53-61, 2013.

[5] D. Bowman, B.R. Mattes, Conductive Fibre Prepared From Ultra-High Molecular Weight Polyaniline for Smart Fabric and Interactive Textile Applications, Synthetic Metals, Volume 154(1-3), pp. 29-32, 2005.

[6] R.I.J. Helmer, M.A. Mestrovic, D. Farrow, S.R. Lucas, W. Spratford, Smart Textiles: Position and Motion Sensing for Sport, Entertainment and Rehabilitation. Advances in Science and Technology, Vol. 60, pp.144$153,2008$.

[7] X. Peng, Z. Guo, T. Dua, W.-R. Yu, A simple anisotropic hyperelastic constitutive model for textile fabrics with application to forming simulation, Composites: Part B, Vol. 52, pp.275-281, 2013.

[8] A.S. Chen, F.L. Matthews, A review of multiaxial/biaxial loading tests for composite materials, Composites, Vol. 24(5), pp. 395-406, 1993. 
[9] D. Lecompte, A. Smits, H. Sol, J. Vantomme, D. Van Hemelrijck, Mixed numerical-experimental technique for orthotropic parameter identification using biaxial tensile tests on cruciform specimens, International Journal of Solids and Structures, Vol. 44, pp. 1643-1656, 2007.

[10] A. Gasser, P. Boisse, S. Hanklar, Mechanical behaviour of dry fabric reinforcements. 3D simulations versus biaxial tests, Computational Materials Science, Vol. 17, pp. 7-20, 2000.

[11] Y. Hanabusaa, H. Takizawab, T. Kuwabarac, Numerical verification of a biaxial tensile test method using a cruciform specimen, Journal of Materials Processing Technology, Vol. 213, pp. 961-970, 2013.

[12] L. Liu, Z. Su, R. Wanga, X. Luo, Material-aware cloth simulation via constrained geometric deformation, Computers \& Graphics, Vol. 37, pp. 21-32, 2013.

[13] J. Lu, C. Zheng, Dynamic cloth simulation by isogeometric analysis, Computer Methods in Applied Mechanics and Engeneering, Vol. 268, pp. 475-493, 2014.

[14] D. Zhang, M.M.F. Yuen, Cloth simulation using multilevel meshes, Computers \& Graphics, Vol. 25 pp. 383, 389, 2001.

[15] C. Santulli, F. Sarasini, J. Tirillò, T. Valente, M. Valente, A.P. Caruso, M. Infantino, E. Nisini, G. Minak, Behaviour of jute cloth/wool felts hybrid laminates, Materials and Design, Vol. 50, pp.309-321, 2013.

[16] C.Z., X. Jin, C.C.L. Wang, J. Feng, Plausible cloth animation using dynamic bending model, Progress in Natural Science, Vol. 18, pp. 879-885, 2008.

[17] J. Kaldor, D.L. James, S. Marschner, Simulating Knitted Cloth at the Yarn Level, Proceedings of SIGGRAPH 2008, Los Angeles, California, August 2008.

[18]J. G. Teng J.G., Chen S.F., Hu J.L., A finite-volume method for deformation analysis of woven fabrics, International Journal for Numerical Methods in Engineering, Vol. 46, pp. 2061-2098, 1999.

[19] Liu X.H., Sze K.Y., A corotational interpolatory model for fabric drape simulation, International Journal for Numerical Methods in Engineering, Vol. 77, pp. 799-823, 2009. 
[20] A. Tabiei, W. Yi, Comparative study of predictive methods for woven fabric composite elastic properties, Composite Structures, Vol. 58, pp. 149-164, 2002.

[21] A. Tabiei, Y. Jiang, Woven fabric composite material model with material nonlinearity for nonlinear finite element simulation, International Journal of Solids and Structures, Vol. 36, pp. 1646-1660, 1999.

[22] A. Tabiei, I. Ivanov, Materially and geometrically non-linear woven composite micro-mechanical model with failure for finite element simulations, International Journal of Non-Linear Mechanics, Vol. 39, pp.175188, 2004.

[23] K. Choi, T. Lo, An energy model of plain knitted fabric. Textile Research Journal, Vol. 73, pp. 739-748, 2003.

[24] K. Choi, S. Tandon, An energy model of yarn bending, Journal of the Textile Institute, Vol. 97, pp. 49-56, 2006.

[25] A. Jaramillo, F. Prieto, P. Boulanger, Deformable part inspection using a spring-mass system, ComputerAided Design, Vol. 45, pp.1128-1137, 2013.

[26] W.-R. Yua, P. Harrisonb, A. Longa, Finite element forming simulation for non-crimp fabrics using a nonorthogonal constitutive equation, Composites: Part A, Vol. 36, pp.1079-1093, 2005.

[27] X. Peng, F. Ding Validation of a non-orthogonal constitutive model for woven composite fabrics via hemispherical stamping simulation, Composites: Part A, Vol. 42, pp. 400-407, 2011. 